\title{
Moderne Aspekte der Aknepathogenese
}

\section{C. Zouboulis}

\section{Modern Aspects of Acne Pathogenesis}

\section{Zusammenfassung}

Akne ist weltweit die häufigste dermatologische Erkrankung. Um eine angesichts variierender klinischer Bilder geeignete Therapie ansetzen zu können, sind gute Kenntnisse über die Pathogenese der Erkrankung nötig. Acne vulgaris ist eine entzündliche Erkrankung, bei deren Entstehung Androgene, PPAR-Liganden, regulierende Neuropeptide und Umweltfaktoren beteiligt sein können. Diese Faktoren unterbrechen den natürlichen zyklischen Prozess im Talgdrüsenfollikel und unterstützen den Übergang von Mikrokomedonen zu Komedonen und entzündlichen Effloreszenzen. Proinflammatorische Lipide, Chemokine und Zytokine übernehmen die Vermittlerrolle bei der Entwicklung von Akneeffloreszenzen. P. acnes ist in diesem Stadium nicht beteiligt, sondern kann später die entzündlichen Phänomene verstärken.

\section{Abstract}

Acne is worldwide the most common skin disease. Suitable therapeutic regimens of the varying clinical phenotypes require good knowledge of the pathogenesis of the illness. Acne vulgaris is an inflammatory disorder at whose emergence androgens, PPAR ligands, regulating neuropeptides and environmental factors are probably involved. These factors interrupt the natural cycling process in the sebaceous gland follicle and support the transition of microcomedones to comedones and inflammatory lesions. Proinflammatory lipids, chemokines and cytokines overtake the role of mediators for the development of acne lesions. P. acnes is not involved in this initial acne stages but can potentate the inflammatory phenomena at a later stage.
Akne ist weltweit die häufigste dermatologische Erkrankung. Rund 70 bis $87 \%$ aller Jugendlichen weisen Akneläsionen auf, 10 bis 30\% davon benötigen eine medikamentöse Therapie [1]. Bei der Mehrzahl der Patienten erfolgt nach der Pubertät eine spontane Rückbildung, davon bei 2 bis $7 \%$ mit erheblicher Narbenbildung. In 10\% der Fälle persistiert die Erkrankung über das 25. Lebensjahr hinaus.

Akne ist nach einer amerikanischen Versorgungsstudie im Jahr 1995 die führende dermatologische Diagnose mit 10,2 Millionen Diagnosen (25,4\% der dermatologischen Diagnosen aller Ärzte)
[2]. In den Jahren 1996 bis 1998 wurden niedergelassene Ärzte in den USA jährlich mehr als 6 Millionen mal aufgrund einer Akne konsultiert. Die Patienten erhielten pro Jahr 6,5 Millionen neue Rezepte mit einer systemischen Antiaknetherapie (Antibiotika oder Isotretinoin) im Wert von über eine Milliarde US-Dollar. Die weltweiten Ausgaben für die topische und systemische Aknetherapie entsprachen 1996 12,6\% der gesamten medikamentösen Kosten zur Behandlung von Hautkrankheiten weltweit. 
Um angesichts variierender klinischer Bilder und unterschiedlicher Manifestationsalter (Abb.1) geeignete Therapieschemata ansetzen zu können, sind gute Kenntnisse über die Pathogenese der Erkrankung sowie Möglichkeiten zur gezielten symptomatischen oder sogar ätiologischen Therapie unabdingbare Voraussetzungen [3]. Traditionell wird behauptet, dass zur Akneentstehung verschiedene Faktoren beitragen, unter anderem eine erhöhte Talgdrüsenaktivität mit Seborrhoe, gestörte follikuläre Differenzierung und verstärkte Verhornung, weiterhin mikrobielle Hyperkolonisation sowie Entzündungsreaktionen mit den entsprechenden immunologischen Abläufen [4].

Die tatsächlichen ätiologischen Faktoren der Erkrankung sollen so vielfältig wie die klinischen Erscheinungsbilder sein [1]. Genetische Studien erbrachten Hinweise auf das Vorhandensein erblicher Faktoren. Bei Frauen üben ein unregelmäßiger Menstruationszyklus und die Schwangerschaft einen Einfluss auf den Verlauf der Akne aus [4]. Bei einigen Patienten sollen Ernährungsfaktoren die Erkrankung beeinflussen. Das Klima einschließlich Luftfeuchtigkeit und UV-Strahlung sowie andere Umweltfaktoren können im Einzelfall eine Rolle spielen. Akne kann durch zahlreiche Medikamente ausgelöst oder verschlechtert werden. Der Einfluss psychischer Faktoren wie Stress auf die Pathogenese der Akne konnte bislang nicht bewiesen werden, sie sind jedoch im Verlauf der Krankheit oftmals von Belang [5]. Aktuelle experimentelle Daten weisen auf die Beteiligung von zirkulierenden stressassoziierten Faktoren (Neuropeptide) bei der Entwicklung von entzündlichen Prozessen im Talgdrüsenfollikel hin [6]. Allerdings sind falsche Behauptungen über die Pathogenese der Erkrankungen nicht nur bei Laien, sondern auch bei fortgeschrittenen Medizinstudenten verbreitet, wie eine Studie aus Australien zeigen konnte [7].

Neue Forschungsergebnisse haben die klassische Sicht zur Aknepathogenese durch die Identifizierung übergeordneter Mechanismen, die zur Entwicklung von Akneefloreszenzen führen, deutlich geändert. Androgene, Hautlipide und regulierende Neuropeptide scheinen an diesem multifaktoriellen Prozess beteiligt zu sein $[1,2]$. Erbliche Faktoren sollen eine wichtige, aber indirekte Rolle bei der Entstehung der Akne spielen [8].

\section{Genetik}

Der positive Zusammenhang von familiärer Häufigkeit und Schweregrad der Akne [9], obligates Auftreten der Akne bei homozygoten Zwillingen [10] und auffällige Häufung bei heterozygoten Zwillingen [11] sind seit längerem bekannt. Allerdings gibt es auch interessante neue Erkenntnisse über eine direkte genetische Assoziation der Akne mit Androgen- und Lipid-assoziierten
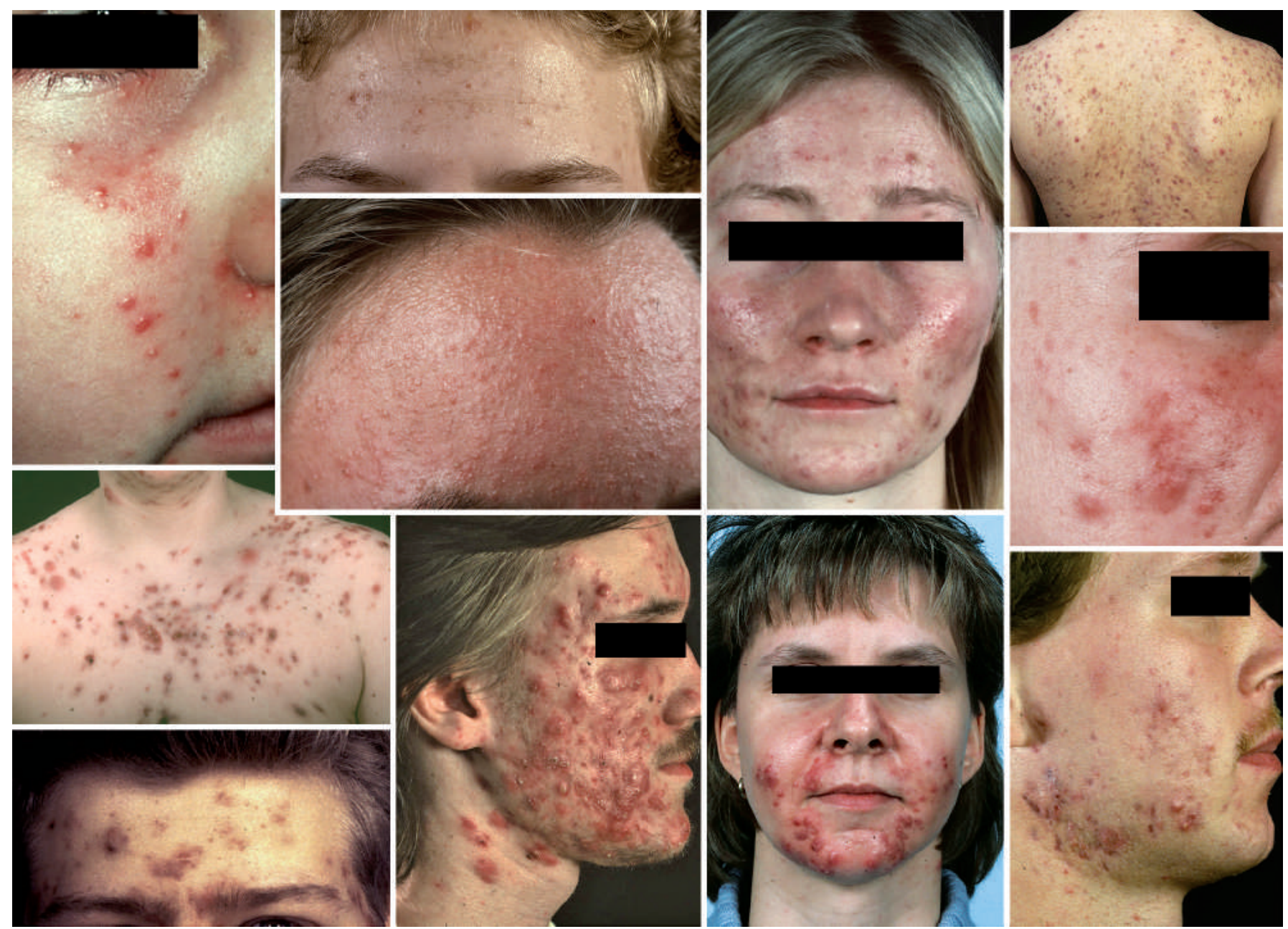

Abb. 1 Die verschiedenen klinischen Gesichter der Acne vulgaris. 
Erkrankungen, beispielsweise a) tritt Neugeborenenakne bei familiärem Hyperandrogenismus auf [12]; b) sind eine abnormale Aktivität der Steroid-21-Hydroxylase sowie CYP21-Genmutationen Akne-assoziiert [13]; c) wurden identische Talgdrüsensekretionsraten bei homozygoten, aber nicht bei heterozygoten Zwillingen gemessen [10]; d) wurden bei Zwillingen mit Akne, aber nicht bei Zwillingen ohne Akne, niedriges Apolipoprotein-A1 im Serum [14], niedriger Gehalt an essentiellen Fettsäuren in den Wachsestern des Talgs und niedrige Werte von epidermalen Acetylceramiden [15] nachgewiesen.

\section{Androgene, Talgsynthese und Akne}

Wie aus klinischen Beobachtungen bekannt, spielen Androgene eine wesentliche Rolle in der Aknepathogenese - sowohl bei der Zunahme des Talgdrüsenvolumens als auch bei der Talgproduktion [16]. Geschlechtsdeterminierende Gene sind an der Entwicklung der Akne beteiligt [17]. Darüber hinaus stimulieren sie die Proliferation der Keratinozyten des Ductus seboglandularis und des Acroinfundibulums $[18,19]$. Akne entwickelt sich schon während der Adrenarche und zwar mit Beginn der Synthesesteigerung von Dehydroepiandrosteronsulfat (DHEA-S), eines Vorstoffes von Testosteron, durch die Nebennierenrinde [20,21]. Bei Hyperandrogenämie bzw. Hyperandrogenismus kommt es zu gesteigerter Talgproduktion und schwerer Akne [22]. Die befallene Haut besitzt eine höhere Androgenrezeptordichte [23] und höhere 5-alpha-Reduktase-Aktivität [24] als die nicht befallene Haut. Antiandrogene reduzieren die Synthese sebozytärer Lipide und verbessern die Akne [25]. Eine androgen-unempfindliche Haut besitzt keine funktionellen Androgenrezeptoren, produziert keinen Talg und entwickelt keine Akne [26].

Die Lipidsynthese benötigt Androgene und PeroxisomProliferation-aktivierende Rezeptoren (PPAR)

In-vitro-Experimente mit talgdrüsenähnlichen Zellen der Ratte (Rosenfield u. Mitarb. [27]) haben gezeigt, dass die sebozytäre Lipidsynthese in Anwesenheit von Androgenen und durch Liganden von PPAR gemeinsam stimuliert wird. Tatsächlich sind menschliche Talgdrüsen sowohl mit Androgenrezeptoren [28] als auch mit PPAR üppig ausgestattet [29-31]. Unter den verschiedenen PPAR-Subtypen sind besonders PPARalpha und PPARgamma bei der Regulation der Lipidsynthese beteiligt [30]. Einer der stärksten natürlichen PPARalpha-Liganden ist das 5-Lipoxygenase-Produkt Leukotrien-B4. Dessen Vorläufer, die Arachidonsäure, stimuliert die sebozytäre Lipogenese bei kultivierten menschlichen Sebozyten [30,32]. Nicht nur die Behandlung mit Antiandrogenen [25], sondern auch die systemische Therapie mit 5-Lipoxygenase-Inhibitoren hemmt die Synthese sebozytärer Lipide und verbessert die Akneeffloreszenzen, wie wir in Pilotstudien mit Zileuton zeigen konnten [33,34]. Interessanterweise sind synthetische Antidiabetika, die PPAR-Liganden Thiazolidinedione und Fibrate, in der Lage, die Talgsekretionsrate bei Diabetikern zu erhöhen [35].

\section{Molekulare Entzuindungskaskade und Startsignal}

Eine Hyperproliferation des follikulären Epithels führt zur Bildung von Mikrokomedonen. Diese stellen die initiale Akneeffloreszenz dar, kommen aber auch in der normal aussehenden Haut vor [36]. Der Talgdrüsenfollikel könnte einem zyklischen Prozess unterliegen, der zu einer natürlichen Resolution der Mikrokomedonen führt [37] (Abb. 2). Diese frühe Stufe der Entwicklung von Akneeffloreszenzen wird mit einer Aktivierung des vaskulären Endothels und mit einer Beteiligung entzündlicher Prozesse assoziiert [38]. Dies bestätigt die Hypothese, dass Akne eine entzündliche Erkrankung ist, die ohne Beteiligung von Bakterien auftreten kann [39]. Die Ergebnisse von Ingham u. Mitarb. [40] weisen in die gleiche Richtung: Sie fanden bioaktives Interleukin (IL)-1 in offenen Aknekomedonen bei unbehandelten Patienten (Abb. 3). Darüber hinaus gab es keine Korrelation zwischen dem Zytokinniveau und der Anzahl follikulärer Mikroorganismen.

Interessanterweise exprimieren gesunde Talgdrüsen zahlreiche Zytokine. In unserem Labor stressten wir Sebozyten in vitro in serumfreiem Medium und konnten IL-1alpha auf mRNS- und Proteinebene nachweisen [41]. Antilla u. Mitarb. [42] zeigten, dass auch normale Talgdrüsen IL-1 exprimieren können, während Boehm u. Mitarb. [43] mRNS von IL-1alpha, IL-1beta und Tumornekrosenfaktor-alpha in normalen Talgdrüsen mittels Insitu-Hybridisierung fanden. Guy u. Mitarb. [44] zeigten, dass IL1alpha ex vivo bei Talgdrüsenfollikelinfundibula eine gesteigerte follikuläre Keratinozytenproliferation induziert.

\section{Unterbrechung des zyklischen Prozesses des Talgdrüisenfollikels}

Eine Überstimulation des präklinischen entzündlichen Prozesses oder ein Fehler der negativen Feedback-Regulation können Hauptgründe für die Unterbrechung des normalen zyklischen Prozesses im Talgdrüsenfollikel und für die Entwicklung einer klinisch relevanten follikulären Entzündung bei Akne sein (Abb. 2). Wie vorher erwähnt, verursachen genetische Faktoren einen Überschuss der Androgenaktivität in der Pubertät, der entzündliche Veränderungen auslösen kann (Abb.4). Neuroendokrine Regulationsmechanismen, follikuläre Bakterien, proinflammatorische sebozytäre Lipide sowie Nahrungslipide und Rauchen wirken dabei wahrscheinlich als Ko-Faktoren, welche die entzündlichen Prozesse verstärken können.

Neuropeptide und klinisch relevante follikuläre Entzüindung

Es gibt gegenwärtige Beweise, dass regulierende Neuropeptide (mit hormoneller und nicht-hormoneller Aktivität) die Entwicklung der klinischen Entzündung bei Akne kontrollieren können [6]. In der Haut von Aknepatienten lassen sich zahlreiche immunreaktive Nervenfasern nachweisen, die Substanz-P exprimieren [45]. Außerdem wird in näher stehenden undifferenzierten Talgdrüsenzellen neutrale Endopeptidase exprimiert. Ex vivo-Versuche demonstrierten, dass Substanz-P eine dosisabhängige Expression der neutralen Endopeptidase in den Talgdrüsen verursachte. Neben der neutralen Endopeptidase exprimieren Talgdrüsenzellen auch andere Ektopeptidasen, nämlich Dipepti- 


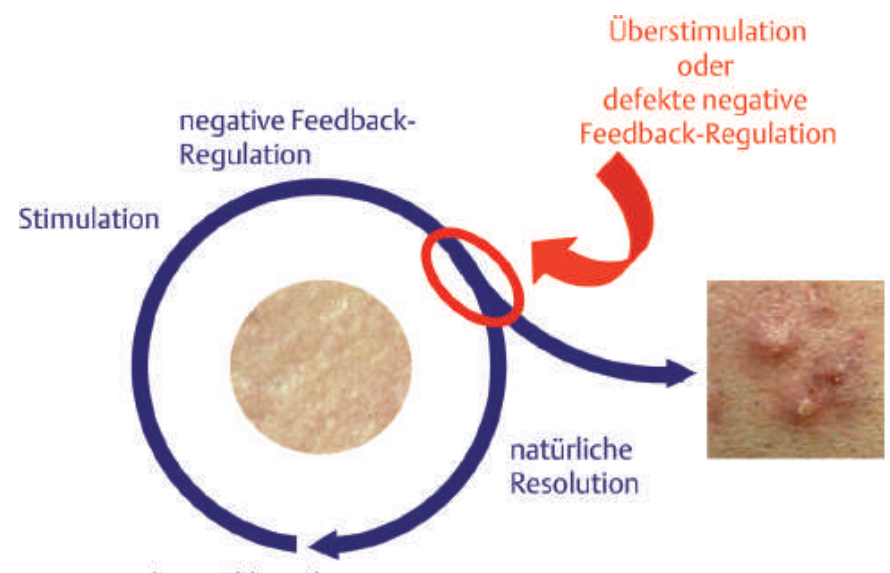

Initiation des präklinischen

Entzündungsprozesses

Abb. 2 Natürlicher zyklischer Prozess im Talgdrüsenfollikel (Mikrokomedo). Unkontrollierte Überstimulation oder Fehler der negativen Feedback-Regulation können zur Entwicklung von klinisch relevanten Akneeffloreszenzen, nämlich Komedonen und entzündlichen Papeln führen (aus [2]).

dylpeptidase-IV (CD26) and Aminopeptidase-N (CD13), deren Hemmung Proliferation, Lipidsynthese und Freisetzung proinflammatorischer Zytokine reguliert [46].

Eine Behandlung von Sebozyten mit IL-1beta führte zu signifikanter Zunahme der IL-8-Freisetzung [47]. Eine Ko-Inkubation der Zellen mit alpha-Melanozyten-stimulierendem Hormon (alpha-MSH) hemmte dosisabhängig die IL-8-Expression [48]. Ferner konnten wir zeigen, dass das Kortikotropin-freisetzende Hormon (CRH) die Synthese von Talgdrüsenlipiden in vitro [47] und die Freisetzung von IL-6 und IL-8 [49] stimuliert, wobei wir in Akne-beteiligter Haut eine erhöhte CRH-Expression nachweisen konnten (Ganceviciene R u. Mitarb., in Vorbereitung). Andererseits fördert Adrenokortikotropes Hormon (ACTH) die Synthese und Freisetzung von adrenalem Dehydroepiandrosteron (DHEA), das eine follikuläre Entzündung stimulieren kann [50]. Diese Befunde weisen auf eine zentrale [51] oder periphere Neuroregula-

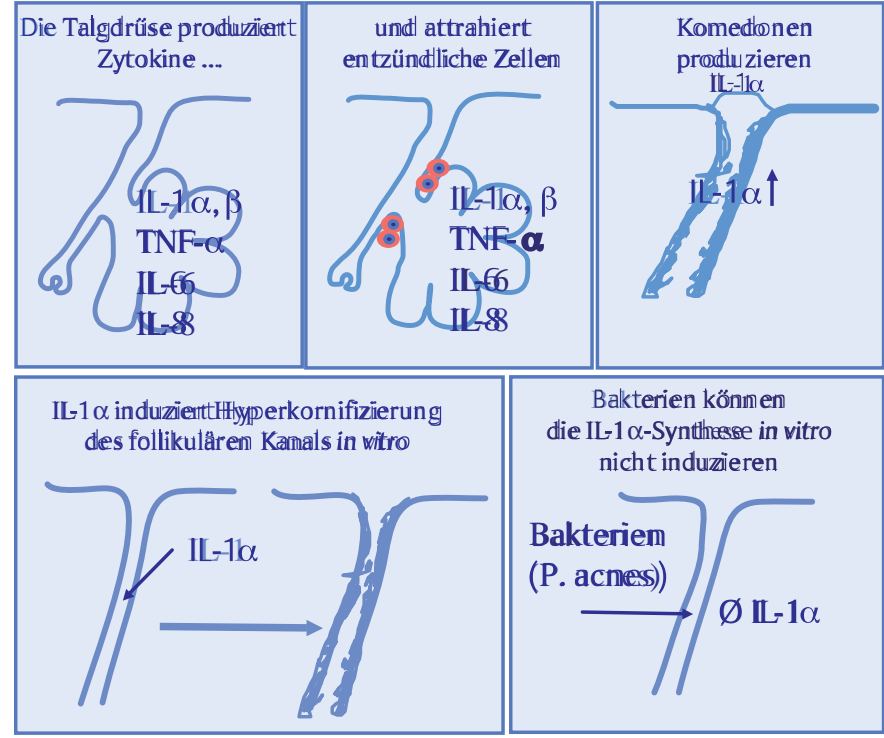

Abb. 3 Entzündung und Talgdrüsenfollikel. Expression proentzündlicher Zytokine in den Talgdrüsenzellen und Versammlung von einzelnen Lymphozyten um den Ductus seboglandularis sind bereits in gesunder Gesichtshaut nachweisbar. In den Komedonen wird eine erhöhte IL-1alpha-Konzentration gemessen, wobei die Behandlung des Acroinfundibulums ex vivo mit IL-1alpha eine Komedonen-ähnliches Bild mit Proliferation der follikulären Keratinozyten verursacht. P. acnes führt weder zur erhöhten IL-1alpha-Synthese noch zu Veränderungen des follikulären Epithels.

tion $[47,52]$ des negativen Feedback-Mechanismus der humanen Talgdrüse hin und verstärken die Hypothese der neurogenen Induktion einer klinischen Entzündung bei Patienten mit Akne.

\section{Lipide und Alkne}

Die lokale Anwendung von Linolsäure über einen Monat konnte eine fast 25-prozentige Reduktion der Mikrokomedonen erreichen [53]. Andererseits stimulieren Arachidonsäure und andere langkettige proinflammatorische Omega-6-Fettsäuren die IL-8-

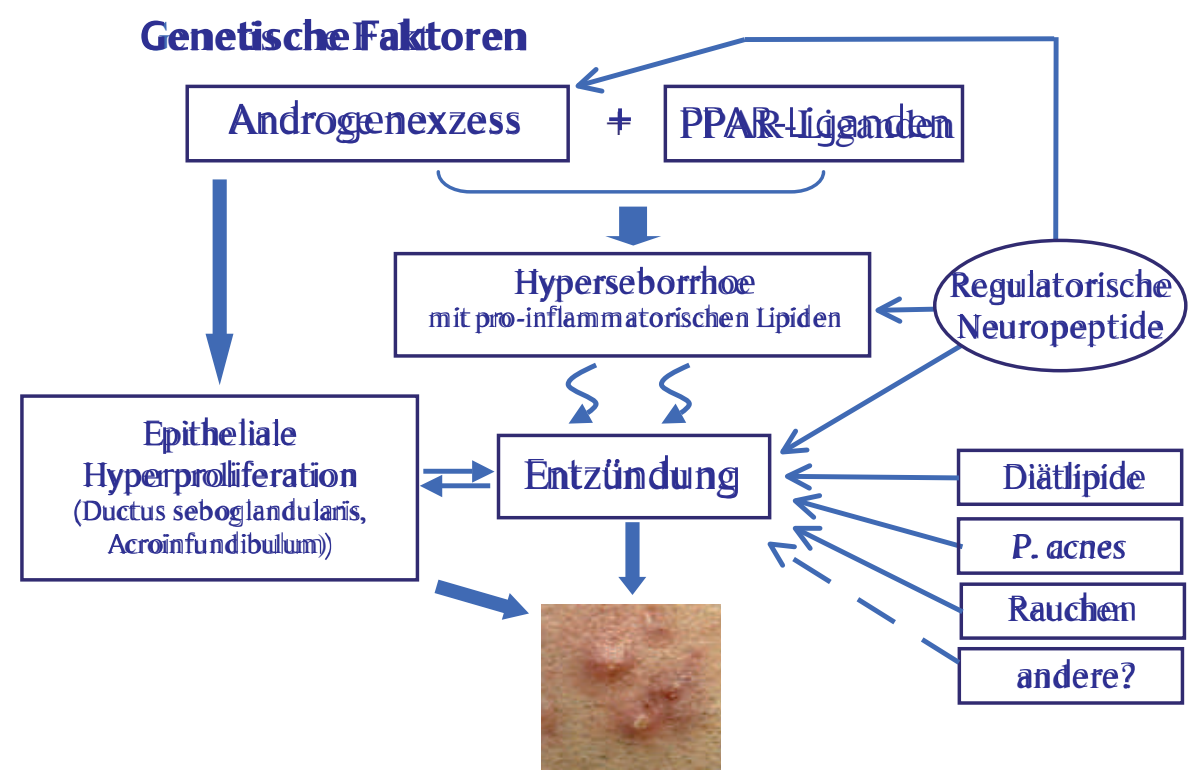

Abb. 4 Moderne Aspekte der Aknepathogenese. Androgene, Liganden der Peroxisom-Proliferation-aktivierenden Rezeptoren (PPAR), regulierende Neuropeptide mit hormoneller und nicht-hormoneller Aktivität und Umweltfaktoren führen zu Hyperseborrhoe, epithelialer Hyperproliferation im Ductus seboglandularis und dem Acroinfundibulum und zur Expression von pro-entzündlichen Chemokinen/Zytokinen, die die Entwicklung von Komedonen und entzündlichen Effloreszenzen stimulieren (aus [2]). 
und IL-6-Synthese [30] sowie die Synthese sebozytärer Lipide [32] in kultivierten menschlichen Sebozyten. Eine Hemmung von Leukotrien-B4 in vivo reduziert die proinflammatorischen Talgdrüsenfettsäuren und damit die Zahl entzündlicher Akneeffloreszenzen $[33,34]$. Wir konnten nachweisen, dass der Arachidonsäuremetabolismus bei Aknepatienten durch eine Aktivierung der 5-Lipoxygenase auf der gesamten Haut erhöht ist, während eine Aktivierung der Ciclooxygenase-2 lediglich bei Akneeffloreszenzen vorliegt [30]. Andererseits ist Ciclooxygenase-2 an der PPARgamma-regulierten Prostaglandin-2-Synthese in humanen Sebozyten beteiligt [54].

Interessanterweise wird bei Eskimos, den Einwohnern der Okinawa-Insel und Chinesen in Zusammenhang mit dem Wechsel ihrer Ernährungsgewohnheiten häufiger Akne beobachtet $[29,55,56]$. Die verwestlichte Ernährung schließt eine niedrige Menge an Omega-3-Fettsäuren und antioxidativen Vitaminen sowie höhere Mengen von proinflammatorischen Omega-6-Fettsäuren ein. Das Verhältnis Omega-6-/Omega-3-Fettsäuren in der westlichen Ernährung beträgt 20:1, im Gegensatz zu einem 1:1-Verhältnis in traditionellen Ernährungsschemata [57].

Insgesamt bleibt die Rolle der Ernährung bei Akne immer noch strittig. Auch wenn Cordell u. Mitarb. [58] berichtet haben, dass es bei den Kitava-Insulanern in Papua-Neuguinea und den AcheJägern in Paraguay keine Aknepatienten gibt [58], lässt sich nicht sagen, ob dies an den Genen oder an der Ernährung liegt [59].

\section{Rauchen und Akne}

Zwischen Akneschweregrad und täglicher Zahl an gerauchten Zigaretten wurde eine klare positive Assoziation nachgewiesen [60]. Allerdings zeigten andere Studien keinen Zusammenhang zwischen Rauchen und Akne [61] oder sogar einen negativen Zusammenhang, wobei Raucher erinnern, weniger oder leichte Akne gehabt zu haben $[62,63]$. Untersuchungen ergaben, dass der Zigarettenrauch hohe Mengen an Arachidonsäure und polyzyklischen aromatischen Kohlenwasserstoffen enthält [64]. Diese initiieren einen Phospholipase-A2-abhängigen Signalweg, der die proentzündliche Wirkung von Arachidonsäure weiter stimulieren kann [65]. Andererseits nehmen Raucher aber auch häufiger eine Nahrung reich an gesättigten und arm an ungesättigten Fetten ein, was zu einer vergleichsweise niedrigeren Konzentration von Linolsäure-Aufnahme bei Rauchern führt [66].

\section{Propionibacterium acnes (P. acnes) und Toll-like-Rezeptoren}

Sowohl Toll-like-Rezeptoren-2 und -4 als auch CD14 werden in menschlichen Monozyten und Keratinozyten exprimiert [67,68]. Die Chemokin-/Zytokin-Synthese wird in Keratinozyten durch Aktivierung des Toll-like-Rezeptors-2 via P. acnes stimuliert, wobei diese Aktivierung P. acnes-Subtyp-abhängig ist [69]. Diese Befunde haben die Diskussion um die Beteiligung von $P$. acnes an der Akne-assoziierten Entzündung erneut angefacht. Jedoch erwies sich $P$. acnes außerstande, die Freisetzung von IL-1 aus menschlichen Keratinozyten in vitro zu stimulieren [70] (Abb. 3). Darüber hinaus werden Toll-like-Rezeptoren-2 und -4 und CD14 auch in Sebozyten exprimiert [71,72]. Die Erkennung von $P$. acnes oder der unspezifischen bakteriellen Antigene Lipopolysaccharide führt zur Induktion der Expression des Enzyms Stearoyl-Coenzym-A-Desaturase in humanen Sebozyten und der Synthese der sebozytären Fettsäuren Palmitoleat (C16:1) und Oleat (C18:1) [73] und des humanen beta-Defensin-2 [74], die eine signifikante antibakterielle Aktivität besitzen. Dieser Mechanismus weist auf die Rolle von P. acnes als komensales Bakterium hin, dass für eine Bereitschaft der endogenen Immunität der Haut gegen pathogene Keime verantwortlich ist.

Deshalb scheint $P$. acnes eher an einem späteren Stadium der Akneentwicklung beteiligt zu sein, wenn es zu bedeutendem follikulärem Wachstum kommt, und nicht an der Initiation der Akneeffloreszenzen. Der erfolgreiche therapeutische Einsatz von Antibiotika bei Akne ist nicht allein auf eine antibakterielle Aktivität zurückzuführen, sondern kann auch als Ausdruck einer paraantibiotischen antientzündlichen Wirkung betrachtet werden

\section{Literatur}

1 Zouboulis CC. Acne: Sebaceous gland action. Clin Dermatol 2004; 22: $360-366$

2 Zouboulis CC, Eady A, Philpott M, Goldsmith LA, Orfanos C, Cunliffe WC, Rosenfield R. What is the pathogenesis of acne? Exp Dermatol 2005; $14: 143-152$

${ }^{3}$ Zouboulis CC. Moderne Aknetherapie. Akt Dermatol 2003; 29: 49-57

${ }^{4}$ Zouboulis CC. Acne: Current aspects on pathology and treatment. Dermatol Experiences 1999; 1: 6-37

${ }^{5}$ Chiu A, Chon SY, Kimball AB. The response of skin disease to stress: changes in the severity of acne vulgaris as affected by examination stress. Arch Dermatol 203; 139: 897-900

${ }^{6}$ Zouboulis CC, Böhm M. Neuroendocrine regulation of sebocytes - a pathogenetic link between stress and acne. Exp Dermatol 2004; 13 (Suppl 4): 31 - 35

${ }^{7}$ Green J, Sinclair RD. Perceptions of acne vulgaris in final year medical student written examination answers. Australas J Dermatol 2001; 42: $98-101$

${ }^{8}$ Herane MI, Ando I. Acne in infancy and acne genetics. Dermatology 2003; 206: 24-28

${ }^{9}$ Goulden V, McGeown $\mathrm{CH}$, Cunliffe WJ. The familial risk of adult acne: a comparison between first-degree relatives of affected and unaffected individuals. Brit J Dermatol 1999; 141: 297- 300

${ }^{10}$ Walton S, Wyatt EH, Cunliffe WJ. Genetic control of sebum excretion and acne - a twin study. Brit J Dermatol 1988; 118: 393 - 396

${ }^{11}$ Kirk KM, Evans DM, Farthing B, Martin NG. Genetic and environmental influences on acne in adolescent twins. Twin Res 2001; 4: 190

12 Bekaert C, Song M, Delvigne A. Acne neonatorum and familial hyperandrogenism. Dermatology 1998; 196: 453 - 454

13 Ostlere LS, Rumsby G, Holownia P, Jacobs HS, Rustin MHA, Honour JW. Carrier status for steroid 21-hydroxylase deficiency is only one factor in the variable phenotype of acne. Clin Endocrinol 1998; 48: 209-215

${ }^{14}$ Bataille V, Snieder H, MacGregor AJ, Sasieni P, Spector TD. The influence of genetics and environmental factors in the pathogenesis of acne: a twin study of acne in women. J Invest Dermatol 2002; 119: 1317 1322

15 Stewart ME, Grahek MO, Cambier LS, Wertz PW, Downing DT. Dilutional effect of increased sebaceous gland activity on the proportion of linoleic acid in sebaceous wax esters and in epidermal acylceramides. J Invest Dermatol 1986; 87: 733 - 736

16 Pochi PE, Strauss JS. Sebaceous gland response in man to the administration of testosterone, $\Delta 4$-androstenedione, and dehydroisoandrosterone. J Invest Dermatol 1969; 52: 32 - 36

${ }^{17}$ Chen W, Yang C-C, Sheu H-M, Seltmann H, Zouboulis CC. Expression of sex-determining genes in human sebaceous glands and their possible roles in pathogenesis of acne. J Eur Acad Dermatol Venereol; in Druck

${ }^{18}$ Cunliffe W, Forster R. Androgen control of the pilosebaceous duct? Brit J Dermatol 1987; 116: 449 
19 Thiboutot D, Knaggs H, Gilliland K, Lin G. Activity of 5-alpha-reductase and 17-beta-hydroxysteroid dehydrogenase in the infrainfundibulum of subjects with and without acne vulgaris. Dermatology 1998; 196: $38-42$

${ }^{20}$ Lucky AW, Biro FM, Huster GA, Leach AD, Morrison JA, Ratterman J. Acne vulgaris in premenarchal girls. Arch Dermatol 1994; 130: 308 314

${ }^{21}$ Stewart ME, Downing DT, Cook JS, Hansen JR, Strauss JS. Sebaceous gland activity and serum dehydroepiandrosterone sulfate levels in boys and girls. Arch Dermatol 1992; 128: 1345 - 1348

${ }^{22}$ Marynick SP, Chakmajian ZH, McCaffree DL, Herdon JH. Androgen excess in cystic acne. N Engl J Med 1983; 308: 981 - 986

${ }^{23}$ Schmidt JB, Spona J, Huber J. Androgen receptor in hirsutism and acne. Gynecol Obstet Invest 1986; 22: 206-211

${ }^{24}$ Thiboutot D, Harris G, Iles V, Cimis G, Gilliland K, Hagari S. Activity of the type 15 alpha-reductase exhibits regional differences in isolated sebaceous glands and whole skin. J Invest Dermatol 1995; 105: 209 214

${ }^{25}$ Zouboulis CC. Therapie der Akne mit Antiandrogenen - Eine evidenzbasierte Übersicht. JDDG 2003; 1: 535-546

${ }^{26}$ Imperato-McGinley J, Gautier T, Cai LQ Yee B, Epstein J, Pochi P. The androgen control of sebum production. Studies of subjects with dihydrotestosterone deficiency and complete androgen insensitivity. J Clin Endocrinol Metabol 1993; 76: 524-528

${ }^{27}$ Rosenfield RL, Deplewski D, Kentsis A, Ciletti N. Mechanisms of androgen induction of sebocyte differentiation. Dermatology 1998; 196: $43-46$

${ }^{28}$ Fimmel S, Saborowski A, Térouanne B, Sultan C, Zouboulis CC. Inhibition of the androgen receptor by antisense oligonucleotides regulates the biological activity of androgens in SZ95 sebocytes. Horm Metab Res; in Druck

${ }^{29}$ Chen W, Yang C-C, Sheu H-M, Seltmann H, Zouboulis CC. Expression of peroxisome proliferator-activated receptor and CCAAT/enhancer binding protein transcription factors in cultured human sebocytes. J Invest Dermatol 2003; 121: $441-447$

${ }^{30}$ Alestas T, Ganceviciene R, Fimmel S, Müller-Decker K, Zouboulis CC. Enzymes involved in the biosynthesis of leukotriene $\mathrm{B}_{4}$ and prostaglandin $E_{2}$ are active in sebaceous glands. J Mol Med 2006; 84: 75-87

${ }^{31}$ Makrantonaki E, Zouboulis CC. Testosterone metabolism to $5 \alpha$-dihydrotestosterone and synthesis of sebaceous lipids is regulated by the peroxisome proliferator-activated receptor ligand linoleic acid in human sebocytes. Brit J Dermatol; in Druck

32 Wróbel A, Seltmann H, Fimmel S, Müller-Decker K, Tsukada M, Bogdanoff B, Mandt N, Blume-Peytavi U, Orfanos CE, Zouboulis CC. Differentiation and apoptosis in human immortalized sebocytes. J Invest Dermatol 2003; 120: 175-181

${ }^{33}$ Zouboulis CC, Nestoris S, Adler YD, Picardo M, Camera E, Orth M, Orfanos CE, Cunliffe WJ. A new concept for acne therapy: a pilot study with zileuton, an oral 5-lipoxygenase inhibitor. Arch Dermatol 2003; 139: $668-670$

${ }^{34}$ Zouboulis CC, Saborowski A, Boschnakow A. Zileuton, an oral 5-lipoxygenase inhibitor, directly reduces sebum production. Dermatology 2005; 210: $36-38$

${ }^{35}$ Trivedi NR, Cong Z, Nelson AM, Albert AJ, Rosamilia LL, Sivarajah S, Gilliland KL, Liu W, Mauger DT, Gabbay RA, Thiboutot DM. Peroxisome proliferator-activated receptors increase human sebum production. J Invest Dermatol; in Druck

${ }^{36}$ Norris JF, Cunliffe WJ. A histological and immunocytochemical study of early acne lesions. Brit J Dermatol 1988; 118: 651 - 659

${ }^{37}$ Cunliffe WJ, Holland DB, Clark SM, Stables GI. Comedogenesis: some new aetiological, clinical and therapeutic strategies. Brit J Dermatol 2000; 142: $1084-1091$

38 Jeremy AHT, Holland DB, Roberts SG, Thomson KF, Cunliffe WJ. Inflammatory events are involved in acne lesion initiation. J Invest Dermatol 2003; $121: 20-27$

${ }^{39}$ Zouboulis CC. Is acne vulgaris a genuine inflammatory disease? Dermatology 2001; 203: 277-279

${ }^{40}$ Ingham E, Eady EA, Goodwin CE, Cove JH, Cunliffe WJ. Pro-inflammatory levels of interleukin-1 alpha-like bioactivity are present in the majority of open comedones in acne vulgaris. J Invest Dermatol 1992; 98: $895-901$

${ }^{41}$ Zouboulis CC, Xia L, Akamatsu H, Seltmann H, Fritsch M, Hornemann S, Ruhl R, Chen W, Nau H, Orfanos CE. The human sebocyte culture model provides new insights into development and management of seborrhoea and acne. Dermatology 1998; 196: 21 - 31
${ }^{42}$ Antilla HS, Reitamo S, Saurat JH. Interleukin 1 immunoreactivity in sebaceous glands. Brit J Dermatol 1992; 127: 585-588

${ }^{43}$ Boehm KD, Yun JK, Strohl KP, Elmets CA. Messenger RNAs for the multifunctional cytokines interleukin-1 alpha, interleukin-1 beta and tumor necrosis factor-alpha are present in adnexal tissues and in dermis of normal human skin. Exp Dermatol 1995; 4: 335-341

${ }^{44}$ Guy R, Green M, Kealey T. Modeling of acne in vitro. J Invest Dermatol 1996; 106: $176-182$

45 Toyoda M, Nakamura M, Makino T, Kagoura M, Morohashi M. Sebaceous glands in acne patients express high levels of neutral endopeptidase. Exp Dermatol 2002; 11: 241 - 247

${ }^{46}$ Thielitz A, Reinhold D, Vetter R, Lendeckel U, Kähne T, Bank U, Helmuth M, Neubert K, Faust J, Hartig R, Wrenger S, Zouboulis CC, Ansorge S, Gollnick H. Inhibitors of dipeptidyl peptidase IV (DP IV, CD26) and aminopeptidase N (APN, CD13) target major pathogenetic steps in acne initiation. J Invest Dermatol; in Druck

47 Zouboulis CC, Seltmann H, Hiroi N, Chen W, Young M, Oeff M, Scherbaum WA, Orfanos CE, McCann SM, Bornstein SR. Corticotropin releasing hormone: an autocrine hormone that promotes lipogenesis in human sebocytes. Proc Natl Acad Sci USA 2002; 99: 7148 - 7153

${ }^{48}$ Böhm M, Schiller M, Stander S, Seltmann H, Li Z, Brzoska T, Metze D, Schiöth HB, Skottner A, Seiffert K, Zouboulis CC, Luger TA. Evidence for expression of melanocortin-1 receptor in human sebocytes in vitro and in situ. J Invest Dermatol 2002; 118: $533-539$

${ }^{49}$ Krause K, Schnitger A, Fimmel S, Glass E, Zouboulis CC. Corticotropinreleasing hormone skin signalling is receptor-mediated and is predominant in the sebaceous glands. Horm Metab Res; in Druck

${ }^{50}$ Alesci S, Bornstein SR. Neuroimmunoregulation of androgens in the adrenal gland and the skin. Horm Res 2000; 54: 281 - 286

${ }^{51}$ Slominski AT, Botchkarev V, Choudhry M, Fazal N, Fechner K, Furkert J, Krause E, Roloff B, Sayeed M, Wei E, Zbytek B, Zipper J, Wortsman J, Paus R. Cutaneous expression of CRH and CRH-R. Is there a ,skin stress response system?" Ann N Y Acad Sci 1999; 885: 287-311

52 Zouboulis CC. The human skin as a hormone target and an endocrine gland. Hormones 2004; 3: 9-26

53 Letawe C, Boone M, Pierard GE. Digital image analysis of the effect of topically applied linoleic acid on acne microcomedones. Clin Exp Dermatol 1998; 23: 56-58

${ }^{54}$ Zhang Q, Seltmann H, Zouboulis CC, Konger RL. Involvement of PPARgamma in oxidative stress-mediated prostaglandin $\mathrm{E}_{2}$ production in SZ95 human sebaceous gland cells. J Invest Dermatol 2006; 126: 42 48

${ }^{55}$ Schaefer O. When the Eskimo comes to town. Nutr Today 1971; 6: 8 16

${ }^{56}$ Steiner PE. Necropsies on Okinawans: anatomic and pathologic observations. Arch Pathol 1946; 42: 359-380

${ }^{57}$ Simopoulos AP. Evolutionary aspects of diet and essential fatty acids. World Rev Nutr Diet 2001; 88: 18 - 27

${ }^{58}$ Cordain L, Lindeberg S, Hurtado M, Hill K, Eaton SB, Brand-Miller J. Acne vulgaris: a disease of Western civilization. Arch Dermatol 2002; 138: $1584-1590$

59 Thiboutot DM, Strauss JS. Diet and acne revisited. Arch Dermatol 2002; 138: 1591 - 1592

${ }^{60}$ Schäfer T, Nienhaus A, Vieluf D, Berger J, Ring J. Epidemiology of acne in the general population: the risk of smoking. Brit J Dermatol 2001; 145: $100-104$

${ }^{61}$ Firooz A, Sarhangnejad R, Davoudi SM, Nassiri-Kashani M. Acne and smoking: is there a relationship? BMC Dermatol 2005; 5: 2

${ }^{62}$ Galobardes B, Davey Smith G, Jeffreys M, Kinra S, McCarron P. Acne in adolescence and cause-specific mortality: lower coronary heart disease but higher prostate cancer mortality: the Glasgow Alumni Cohort Study. Am J Epidemiol 2005; 161: 1094-1101

${ }^{63} \mathrm{Klaz}$ I, Kochba I, Shohat T, Zarka S, Brenner S. Severe acne vulgaris and tobacco smoking in young men. J Invest Dermatol; in Druck

${ }^{64}$ Tithof PK, Elgayyar M, Cho Y, Guan W, Fisher AB, Peters-Golden M. Polycyclic aromatic hydrocarbons present in cigarette smoke cause endothelial cell apoptosis by a phospholipase A2-dependent mechanism. FASEB J 2002; 16: 1463 - 1464

${ }^{65}$ Zouboulis CC. Human skin: An independent peripheral endocrine organ. Horm Res 2000; 54: 230-242

${ }^{66}$ Thompson RL, Pyke S, Scott EA, Thompson SG, Wood DA. Cigarette smoking, polyunsaturated fats, and coronary heart disease. Ann N Y Acad Sci 1993; 686: 130-138

${ }^{67}$ Kim J, Ochoa MT, Krutzik SR, Takeuchi O, Uematsu S, Legaspi AJ, Brightbill HD, Holland D, Cunliffe WJ, Akira S, Sieling PA, Godowski 
PJ, Modlin RL. Activation of toll-like receptor 2 in acne triggers inflammatory cytokine responses. J Immunol 2002; 169: 1535 - 1541

68 Pivarcsi A, Bodai L, Rethi B, Kenderessy-Szabo A, Koreck A, Szell M, Beer Z, Bata-Csorgoo Z, Magocsi M, Rajnavolgyi E, Dobozy A, Kemeny L. Expression and function of Toll-like receptors 2 and 4 in human keratinocytes. Int Immunol 2003; 15: 721 - 730

${ }^{69}$ Ingham E, Walters CE, Eady EA, Cove JH, Kearney JN, Cunliffe WJ. Inflammation in acne vulgaris: failure of skin micro-organisms to modulate keratinocyte interleukin 1 alpha production in vitro. Dermatology 1998; 196: 86-88

${ }^{70}$ Nagy I, Pivarcsi A, Koreck A, Szell M, Urban E, Kemény L. Distinct strains of Propionibacterium acnes induces selective human $\beta$-defensin-2 and interleukin-8 expression in human keratinocytes through Toll-like receptors. J Invest Dermatol 2005; 124: 931 - 938

${ }^{71}$ Oeff KM, Seltmann H, Hakiy NH, Bogdanoff B, Nastos A, Walters RJ, Fimmel S, Bornstein SR, Zouboulis CC. Differential modulation of Tolllike receptor 2 and 4 expression in human sebocytes. J Invest Dermatol 2002; 119: 319

72 Seltmann H, Zouboulis CC. Human sebocytes express CD14 molecules and their IL8 production is induced by both CD14-dependent and independent pathways. J Invest Dermatol 2001; 117: 804

${ }^{73}$ Georgel P, Crozat K, Lauth X, Makrantonaki E, Seltmann H, Sovath S, Hoebe K, Du X, Rutschmann S, Jiang Z, Bigby T, Nizet V, Zouboulis CC, Beutler B. A TLR2-responsive lipid effector pathway protects mammals against Gram-positive bacterial skin infections. Infect Immun 2005; 73: $4512-4521$

${ }^{74}$ Nagy I, Pivarcsi A, Kis K, Koreck A, Bodai L, McDowell A, Seltmann H, Patrick S, Zouboulis CC, Kemény L. Propionibacterium acnes and LPS induce the expression of antibacterial peptides and proinflammatory cytokines/chemokines in human sebocytes. Microbes Infect; in Druck

\section{Preisausschreibung}

Die Berliner Stiftung für Dermatologie schreibt erneut für das Jahr 2006 ihren mit $\mathbf{1 0 0 0 0} €$ dotierten Forschungspreis aus. Der Preis wird an jüngere Wissenschaftler vergeben, die sich mit der Erforschung der Hautkrankheiten und ihrer Behandlung befassen, und auf nationaler und internationaler Ebene herausragende Verdienste erworben haben. Vorschläge für geeignete Kandidaten mit kurzer Erläuterung und Begründung sind schriftlich bis zum 30. 8. 2006 an die Geschäftsstelle der Stiftung zu richten, z.H. Frau K. Kohler, Dresdner Bank AG, Pariser Platz 6, 10877 Berlin.

Die Entscheidung wird vom Kuratorium der Stiftung bestehend aus Prof. Ch. Geilen, Prof. S. Goerdt, Prof. H. Gollnick, Herr K. von der Heyde, Frau K. Kohler, Prof. h.c. C. E. Orfanos, Prof. J. Simon und Prof. Th. Trautner spätestens bis zum 31.12. 2006 getroffen. Die Preisverleihung erfolgt anlässlich des Jahres-Symposiums der Stiftung im ersten Halbjahr 2007. Die Entscheidung des Kuratoriums ist rechtlich unanfechtbar.

Weitere Informationen zur Stiftung sind über die Geschäftsstelle bzw. über das Internet www.stiftung-dermatologie.de zu erhalten.

\section{Preisverleihung}

Der Hermal Förderpreis Dermatologie 2006 wurde Frau Dr. med. Evgenia Makrantonaki, Hautklinik und Immunologisches Zentrum, Städtisches Kinikum Dessau (Chefarzt: Prof. Dr. Ch. C. Zouboulis) und Labor für Biogerontologie, Dermato-Pharmakologie und Dermato-Endokrinologie (Leiter: Prof. Dr. Ch. C. Zouboulis), Institut für Klinische Pharmakologie und Toxikologie, Charité Universitätsmedizin Berlin, Campus Benjamin Franklin (Direktor: Prof. Dr. M. Paul) für ihre Arbeit „Age-specific hormonal decline is accompanied by transcriptional changes in human sebocytes in vitro" verliehen.

Die Arbeit wurde im Rahmen des explorativen Projektes „Genetische Ätiologie der humanen Langlebigkeit“ des Nationalen Genom-Forschungs-Netzes II (Koordinatoren: Prof. Dr. S. Schreiber, Kiel; Prof. Dr. Ch. C. Zouboulis, Dessau/Berlin) in Kooperation mit dem Max-Planck-Institut für Molekulare Genetik in Berlin (Direktor: Prof. Dr. H. Lehrach, Arbeitsgruppen Dr. J. Adjaye, Dr. R. Herwig) durchgeführt und wurde zur Veröffentlichung im Journal „Aging Cell“ angenommen.

Frau Dr. Makrantonaki hat 2005 ihre Dissertation auf dem Gebiet der hormonellen Alterung an der Freien Universität Berlin mit der Note „summa cum laude“ abgeschlossen. 